\title{
GAME THEORETIC NEGOTIATION STRATEGY FOR VIRTUAL ENTERPRISE WITH MULTIAGENT SYSTEMS
}

\author{
Toshiya Kaihara and Susumu Fujii \\ Kobe University, Department of Computer and Systems Engineering \\ kaihara@cs.kobe-u.ac.jp \\ $J A P A N$
}

\begin{abstract}
Nowadays, Virtual Enterprise (VE) is an important paradigm of business management in agile manufacturing environment. Clearly, there is a need for a mechanism through which these different finctions can be integrated together. In this paper, we focus on negotiation process in VE formation to clarify its effective management. Each enterprise in $V E$ is defined as agent with multiutilities, and a framework of multi-agent programming with marketing science and $N$-person game theoretic approach is newly proposed as negotiation algorithm amongst the agents. We develop a computer simulation model to farm $V E$ through multiple negotiations amongst several potential members in the negotiation domain, and finally clarify the formation dynamism with the negotiation process.
\end{abstract}

\section{INTRODUCTION}

Nowadays, Virtual Enterprise (VE) is an important paradigm of business management in agile manufacturing environment [1]. VE exists in both service and manufacturing organizations, although the complexity of the each enterprise may vary greatly from industry to industry. Traditionally, marketing, distribution, planning, manufacturing, and the purchasing organizations operated independently. These organizations have their own objectives and these are often conflicting. The result of these factors is that there is not a single, integrated plan for the organization - there were as many plans as businesses. Clearly, there is a need for a mechanism through which these different functions can be integrated together.

In this paper, we focus on negotiation process in VE formation to clarify its effective management. Each enterprise in VE is defined as agent, and a framework of multi-agent programming with marketing science and game theoretic approach is newly proposed as negotiation algorithm amongst the agents. Each unit is defined as agent in our VE model, and their decision makings are formulated as marketing science models [2] and $\mathrm{N}$-person game theoretic methodology [3].

We firstly classify business model into three types, such as vertically integrated business model, horizontally specialised business model and hybrid business model. Then we propose CNP based negotiation protocol amongst enterprises with marketing science models, such as lexicographic model and Maximum Likelihood Hierarchical (MLH) model, and N-person game theoretic approach. CNP models 
transfer of control in a distributed system with the metaphor of negotiation among autonomous intelligent beings [4]. CNP consists of a set of nodes that negotiate with one another through a set of message. Nodes generally represent the distributed computing resources to be managed, correspond to "enterprises" in this paper. Marketing science based negotiation is applied into the vertically integrated business model considering a realistic enterprise management strategy. The horizontally specialised business model includes $\mathrm{N}$-person game theoretic negotiations to realise the coordination amongst enterprises in the same business segment. We develop a computer simulation model to form VE through multiple negotiations amongst several potential members in the negotiation domain, and finally clarify the formation dynamism with the negotiation process.

\section{BUSINESS MODEL IN VE}

\subsection{VE Concept}

Virtual enterprises are defined as "agile" enterprises, i.e. as enterprises with integration and reconfiguration capability in useful time, integrated from independent enterprises, primitive or complex, with the aim of taking profit from a specific market opportunity. After the conclusion of that opportunity, the virtual enterprise dissolves and a new virtual enterprise is integrated in order to achieve the necessary competitiveness to respond to another market opportunity [2].

The knowledge and physical resources associated to the development and production of most of today's products often exceed what a single firm is able to accomplish. The new production enterprise is a network that shares experience, knowledge and capabilities - it is critical in this new environment for a manufacturing company to be able to efficiently tap these knowledge and information networks.

The organisational challenge of partitioning tasks amongst partners is of main concern, and can determine the success or failure of a project in the distributed manufacturing environment. They can fit and take advantage of the different competencies in VE, and that leads to their coordination and reconfigurability in order to keep alignment with the market requirements,

Faced to the requirements of competitiveness that the current environment is demanding, enterprises are expected to present at least the following characteristics:

- Fast reconfigurability or adaptability: the ability of fast change face to the unpredictable changes in the environment / market, implying substitution of resources (transition to a new A/V E instantiation)

- Evolutionary capability: the ability to learn with history.

Needless to say, it is very important and difficult activity in forming a virtual enterprise to select appropriate business partners, i.e. partnering, because each enterprise considers not only pursuing its profit but also sharing the risk to join the virtual enterprise. The partnering is described as coordination activity amongst the enterprises, and some sophisticated coordination mechanism is required to realise efficient interactions.

The development of coordination mechanism in computer science can be found in the area of workflow management system, computer supported cooperative work 
(CSCW), and multi-agent systems. The area of multi-agent systems, especially when involving intelligent autonomous agents, has been discussing coordination issues and supporting mechanism [5][6][7]. The interaction capability, both amongst agents and between agents and their environment, is one of the basic characteristics of an agent. In this paper we focus on the contract net protocol (CNP), that is one of the mechanism coming from the early works on multi-agent systems [5], as the coordination and negotiation mechanism amongst business units in VE.

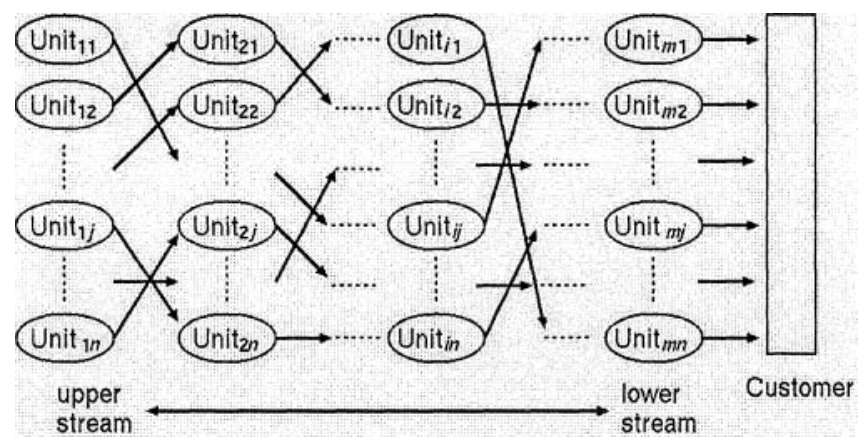

Figure 1 VE model

Figure 1 shows the assumed VE model in this paper. We call an enterprise as unit, and there exist $m$ layers, which have $m_{n}$ units in the VE model. The lowest level corresponds to consumers who can create original task requests to the VE. As the layer number, $m$, increases, we describe it 'lower' based on the product flow order in this paper.

At first, the customer dispatches new order to all the units in layer $m$, and then several units, which are satisfied with the order, responds and circulates the order toward upper units in the VE model. Finally a VE with single supply chain will be established for the order as a consequence of their negotiations through all the layers.

\subsection{Business Model in VE}

As described in the previous section, there exist many business models in VE environment. We classify three types of business models in this study, such as vertically integrated business model, horizontally specialised business model and hybrid business model.

Generally we can observe vertical business models in traditional industries. In this business model the manufacturing business processes top-down as well as bottom-up from the process requirements, resulting in an integrated approach to overall business requirements. We will call this business model, which is based on end-to-end proprietary solutions that lock a customer to a manufacturer, a "vertically integrated business models". Each unit at the same layer in figure 1 never tries to cooperate in our vertically integrated business model, because each unit is keen to find its appropriate partner just in vertical directions. For example, Unit $t_{i j}$ tries to find an alliance unit at each neighbouring layer: layer $(i-1)$ and $(i+1)$ in figure 1 . Consequently only one SC can be formed in this business model. We introduce 
consumer's behaviour in marketing science into unit behaviour, and that makes our VE model more practical.

On the other hand, enterprise relationships may represent a forerunning pattern of the learning alliance, whereby ongoing close interaction of horizontal alliance partners at single level or multiple hierarchical levels can be used to facilitate the mutual accumulation of superior organizational capabilities within the alliance firms. We will call this business model a "horizontally specialised business model". All the units at the same layer try to cooperate to maximise their profit in total in our horizontally specialised business model. They behave as if they are under joint management, and we can see a kind of this style of management in industrial cluster in Japan [8]. We apply $\mathrm{N}$-persons game theory to formulate their behaviour in cooperation, because it describes a decision-making process to find their equilibrium solution in social manner.

Finally hybrid business model combines above-mentioned 2 business models. There exists a parent-child relationship amongst units in VE, and parent company has advantageous in their contract. Units in the child companies try to keep their harmony as a group sharing a common destiny in their management. We apply an alliance strategy based on cooperative game theory in this model.

\section{AGENT DEFINITIONS}

\subsection{Unit Structure}

Each unit (enterprise) is defined as agent in our VE model, and its structure is described in figure 2. We adopt CNP as the coordination and negotiation mechanism amongst the units. CNP models transfer of control in a distributed system with the metaphor of negotiation among autonomous intelligent beings. CNP consists of a set of nodes that negotiate with one another through a set of message. Nodes generally represent the distributed computing resources to be managed, correspond to "units" in this paper.

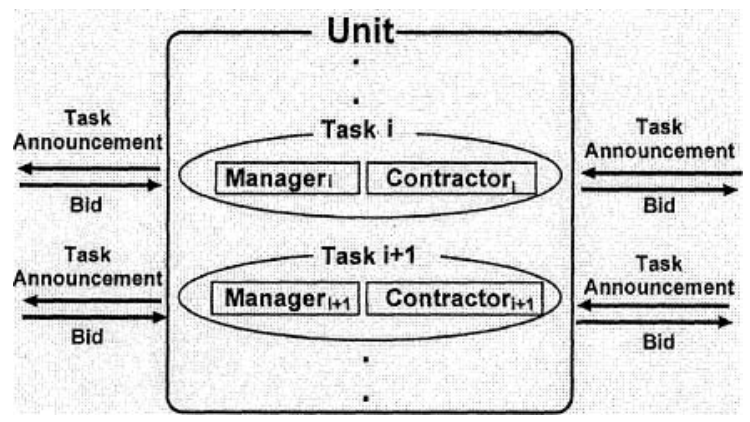

Figure 2 Unit structure

An agent (-unit) can act both as a manager and a contractor of a delivery sets. When a unit receives new order ( $=$ task announcement) $i$, it creates a contractor $/$ manager set (Manager $i$ / Contractor $i$ ) for the task inside. Manager $i$ creates a new order towards the higher units to secure the contract with the lower layer. 


\subsection{Negotiation Algorithm}

Negotiation steps according to agent roles are described as follows:

Manager (in layer $x$ )

Step M1: Create a new task based on the received bid information.

Step M2: Task announcement (TA) to the lower units.

Step M3: After the bidding period expired, check all the acquired bids according to its standard. If there exists no bid to select, go to M4. Otherwise go to M5.

Step M4: Modify the task and go to M2.

Step M5: Select the task and send reward (Reward) to the corresponding unit.

\section{Contractor (in layer $x-1$ )}

Step C1: Evaluate the received TA, and if satisfied, then create an estimated bid. Otherwise wait next TA.

Step C2: Send the bid to the Manager.

Step C3: If the contractor can get the contract, then request the manager in it to create TA to layer $(x-2)$.

We prepare several parameters to define unit behaviours as follows:

$P_{T A}^{i j}:$ TA price of Unit i $_{i j} \quad Q_{T A}^{i j}:$ TA quantity of Unit ${ }_{i j}$

$L_{T A}^{i j}$ : TA lead time of Unit ${ }_{i j} \quad Q_{B I D}^{(i+1) j}$ : Bid quantity of Unit ${ }_{(i+1) j}$

$L_{B I D}^{(i+1) j}$ : Bid lead time of Unit ${ }_{(i+1) j} P_{B I D}^{i j}$ : Bid price of Unit ${ }_{i j}$

$Q_{B I D}^{i j}:$ Bid quantity of Unit ${ }_{i j} \quad L_{B I D}^{i j}$ : Bid lead time of Unit ${ }_{i j}$

$P_{T A}^{(i+1) j}$ : TA price of Unit ${ }_{(i+1) j} \quad Q_{T A}^{(i+1) j}$ : TA quantity of Unit ${ }_{(i+1) j}$

$L_{T A}^{(i+1) j}$ : TA lead time of Unit ${ }_{(i+1) j} \cos t_{i j}$ : process cost of Unit $t_{i j}$ per product

profit $_{i j}$ : profit rate of Unit ${ }_{i j}$ processingtime $_{i j}$ : process time of Unit ${ }_{i j}$ per product

procuretima $_{i j}$ : estimated procure time of $\mathrm{Unit}_{i j}$ per product

Unit formulation in each business model is described in the following sections.

\subsection{Vertically Integrated VE Model}

CNP is just only the skeleton in the negotiation algorithm, and it is necessarily to define how to select the appropriate bid (Step M5) to establish profitable contract. Marketing science based negotiation is applied into the managers' decision makings to realise sophisticated bid selection based on consumers' behaviours in the vertically integrated business model.

First of all, each attribute of manager $_{i j}$ in TA producing process (Step M1) is as follows:

$$
\begin{aligned}
& P_{T A}^{i j}=\cos t_{i j} \times\left(1-\text { profit }_{i j}\right) \\
& Q_{T A}^{i j}=Q_{B I D}^{(i+1) j} \\
& L_{T A}^{i j}=L_{B I D}^{(i+1) j}-Q_{B I D}^{(i+1) j} \times \text { processing time }_{i j}
\end{aligned}
$$

Then contractor ${ }_{i j}$ tries to create its bid (Step C1) against TA from manager in layer $(i+1)$ by the following equations:

$$
\begin{aligned}
& P_{B I D}^{i j}=\cos t_{i j} \times\left(1+\text { profit }_{i j} / 2\right) \\
& Q_{B I D}^{i j}=Q_{T A}^{(i+1) j} \times\left(1+\text { plus }_{i j}\right)
\end{aligned}
$$




$$
L_{B I D}^{i j}=Q_{B I D}^{i j} \times\left(\text { processing time }_{i j}+\text { procuretime }_{i j}\right)
$$

Marketing science approach is applied in the bid selection mechanism (Step M5) in the vertically integrated VE model. We consider 2 types of marketing science model, named lexicographic model and MLH model [3]. Bid selection mechanism is described in each model as follows:

Lexicographic model:

Step L1: Set priorities on all the attributes.

Step L2: Select a bid with the highest value in the attribute. In case of a tie, move to the next attribute and check the highest value. This routine is continued until only one bid is selected.

MLH model:

Step MLH1: Set priorities on all the attributes.

Step MLH2: Standardise the highest attribute with the following equations:

$$
\widetilde{z}_{i j}=\frac{\text { (highest } \left.z_{i j} \text { amongst unselected } j \quad\right)-z_{i j}}{\text { highest } z_{i j} \text { amongst unselected } j}
$$

where $z_{i j}$ : evaluation value of task $j$ on $i$ th attribute

$$
\widetilde{z}_{i j}: \text { standardised } z_{i j}
$$

Step MLH3: If $\widetilde{z}_{i j}$ is within the tolerable amount (i.e. tolerance error: $\tau_{i} \%$ ), then keep this attributes for the calculation in the next step.

Step MLH4: After all the attributes are evaluated, then the preference index $\mathrm{Vj}$ of task $\mathrm{j}$ is calculated:

$$
V_{j}=\left(\tau_{1}-\widetilde{z}_{1 j}\right) \times\left(\tau_{2}-\widetilde{z}_{2 j}\right) \times \cdots \times\left(\tau_{i}-\widetilde{z}_{i j}\right)
$$

Finally selection probability of task $\mathrm{k}_{j}$ is calculated by the following equation:

$$
P_{j}=V_{j} / \sum_{k=1}^{n} V_{k}
$$

\subsection{Horizontally Specialised VE Model}

The horizontally specialised business model includes $\mathrm{N}$-person game theoretic negotiations to realise the coordination amongst enterprises in the same business segment. In this model we consider there is a coordinator to manage the negotiation process amongst all the enterprises (i.e. contractors) in each layer. The coordinator in layer $i$ manages all the units in the layer. It receives all TAs form lower layer $(i+1)$ on behalf of coordinators in the layer $i$, and replies the manager. $N$-person cooperative game approach is applied to share its profit amongst all the units in the layer based on their attributes. So it is regarded as a kind of joint order organisation.

Firstly, TA producing process (Step M1) is in common to the vertically integrated business model. Then the Bidding process is automatically replied based on the received TA, unless it yields no profit in any cooperation of contractor units (Step C2). So the attributes in the bid are defined as follows:

$$
\begin{aligned}
& P_{B I D}^{i j}=P_{T A}^{(i+1) j} \\
& Q_{B I D}^{i j}=Q_{T A}^{(i+1) j}
\end{aligned}
$$




$$
L_{B I D}^{i j}=L_{T A}^{(i+1) j}
$$

Finally the order sharing process is formulated with axioms called Sharpley value. We applied the Shapley value because our VE cooperation model doesn't always satisfy super-additivity condition. The Shapley value describes one approach to the fair allocation of gains obtained by cooperation among several actors even in non super-additivity condition. The setup is as follows: a coalition of actors cooperates, and obtains a certain overall gain from that cooperation. Since some actors may contribute more to the coalition than others, the question arises how to fairly distribute the gains among the actors, in other words, how important is each actor to the overall operation, and what payoff can they reasonably expect? The Shapley value is one way to distribute the total gains to the actors, assuming that they all collaborate.

At first we define characteristic function $(V(S))$ as follows:

$$
V(S)=U_{\text {profit }}(S) \times U_{\text {leadime }}(S)
$$

where $U_{\text {profit }}(S)$ : profit in cooperation $S, \quad U_{\text {leadtime }}(S)$ : Boolean of lead time constraint (1: OK, $0: \mathrm{NG})$

Then the task is divided under the cooperation $S^{\prime}$, which maximises $V(S)$. The contribution of unit $i$ in cooperation $S$ is calculated as follows:

$$
\phi_{i}=\sum_{S: i \in S \subseteq N} \frac{(|S|-1) !(n-|S|) !}{n !}(v(S)-v(S \backslash\{i\}))
$$

where $N$ : a set of all units, $v$ : all the profit in cooperation $S,|S|:$ the number of members in cooperation $S,(S \backslash\{i\})$ : cooperation without unit $i$

Then naturally the following equation is acquired:

$$
\sum_{i \in N} \phi_{i}(v)=v(N)
$$

So the profit, which gained in the coordinator, is divided into each unit in the layer according to the Sharpley value in (14).

\subsection{Hybrid VE Model}

Hybrid business model combines above-mentioned 2 business models. There exists a parent-child relationship amongst units in VE, and parent company has advantageous in their contract, such as in automobile or space industries. Units in the child companies try to keep their harmony as a group in their management.

TA producing process (Step M1) has also no difference in above-mentioned 2 business models. Then contractor ${ }_{i j}$ creates its bid (Step C1) against TA from manager in layer $(i+1)$ by the following equations:

$$
\begin{aligned}
& P_{B I D}^{i j}=\cos t_{i j}\left(1+\text { profit }_{i j}\right) \\
& Q_{B I D}^{i j}=Q_{T A}^{(i+1) j} / N_{i} \\
& L_{B I D}^{i j}=Q_{T A}^{i j} \times\left(\text { processing time }_{i j}+\text { procuretim }_{i j}\right)
\end{aligned}
$$

where $N$ : the number of cooperate units in layer $i$

Finally the bid selection process by manager (Step M5) is formulated by the cooperative game theory under super-additivity condition. We define characteristic function $(V(S))$ as follows: 


$$
\begin{aligned}
& V(S)=U_{\text {profit }}^{\prime}(S) \times U_{\text {leadtime }}(S) \\
& \text { where } U_{\text {proffit }}^{\prime}(S) \text { : manager's profit in cooperation } S
\end{aligned}
$$

Then the characteristic function values in all the received bids are calculated and the manager finally selects the bid with the highest value amongst them.

\section{EXPERIMENTAL MODEL}

We have developed a computer simulation model to analyse VE formation dynamism through multiple negotiations amongst several potential members in the negotiation domain. Although many experiments have done in this study, only a part of our analytic results is explained in this paper due to page limitation. The simulation parameters are shown in table 1.

\begin{tabular}{|c|c|c|c|c|c|c|c|c|c|}
\hline $\mathrm{m}$ & $\mathrm{n}$ & $\tau_{i}$ & $\operatorname{cost}_{0 j}$ & $\operatorname{cost}_{1 j}$ & $\operatorname{cost}_{2 j}$ & profit $_{\text {ij }}$ & $\overline{\text { plus }_{\mathrm{ij}}}$ & $\begin{array}{l}\text { Processing- } \\
\text { time }_{\mathrm{ij}}\end{array}$ & $\begin{array}{l}\text { Procure- } \\
\text { time }_{i j}\end{array}$ \\
\hline 3 & 3,10 & 0.4 & $\begin{array}{l}15- \\
25\end{array}$ & $\begin{array}{l}45- \\
55\end{array}$ & $\begin{array}{l}85- \\
95\end{array}$ & $\begin{array}{l}0.17- \\
0.23\end{array}$ & $\begin{array}{l}0.08- \\
0.12\end{array}$ & $\begin{array}{l}0.08 \\
0.12\end{array}$ & $\begin{array}{l}0.10 \\
0.20\end{array}$ \\
\hline
\end{tabular}

Table 1 Experimental parameters

The performances of finally acquired VE in three business models are compared in Table 2 (Model 1: vertically integrated business model), Table 3 (Model 2: horizontally specialised business model) and Table 4 (Model 3: hybrid model). We tried to analyse the VE robustness of each business model against "due date change" and "production volume change" in this experiment, and 500 trials are examined in each business model. The default due date and production volume are set to 30 and 100 , respectively, and $(0.5,1.0)$, for example, means due date is shorten to $1 / 2(0.5)$ and production volume is equivalent $(1.0)$ to the default in these tables. Only the results of lexicographic model are described in Table 2.

The following points have been observed in those experiments:

- Any models don't satisfy lead time $(15.00)$ in $(0.5,1.5)$, because the requirement change is too heavy to handle.

- Lead time is satisfied at Model 2 and 3 both in $(0.5,1.0)$ and $(1.0,1.5)$. These relatively slight changes are manageable in those business models except Model 1.

- Total profit is the highest at Model 1, because only the contributed units can take direct profit. Additionally stock is the least at this model.

- Model 3 attains the shortest lead time in most cases.

These results have been summarised as follows:

- It is difficult for vertically integrated business model to adapt due date change and production volume change, because it doesn't include any unit cooperation mechanisms. However, it performs best in profit, and this model is efficient in case there are enough margins in lead time (i.e. stable situations).

- Horizontally specialised business model is robust against due date change and production volume change, although the profit is less than vertically 
integrated business model. This business model is suitable to agile manufacturing situations with autonomous cooperation alliance.

- Hybrid model takes a middle position between vertically integrated business model and horizontally specialised business model. Although it takes advantages of both models, it has been observed that the shared profit is inclined into lower layer units in SC.

Table 2 Experimental results of vertically integrated business model (Model 1)

\begin{tabular}{|l|l|l|l|l|}
\hline \multirow{2}{*}{} & $(1.0,1.0)$ & $(0.5,1.0)$ & \\
\cline { 2 - 5 } & Ave. & Std. Dev. & Ave. & Std. Dev. \\
\hline Profit & 8076.05 & 947.00 & 8076.05 & 947.00 \\
\hline Stock(WIP) & 21.14 & 2.50 & 21.14 & 2.50 \\
\hline Lead time & 24.10 & 2.82 & 24.10 & 2.82 \\
\hline & $(1.0,1.5)$ & \multicolumn{3}{|l}{} \\
\cline { 2 - 5 } & Ave. & Std. Dev. & Ave. & Std. Dev. \\
\hline Profit & 12098.30 & 1413.53 & 12098.30 & 1413.53 \\
\hline Stock(WIP) & 31.64 & 3.79 & 31.64 & 3.79 \\
\hline Lead time & 36.10 & 4.21 & 36.10 & 4.21 \\
\hline
\end{tabular}

Table 3 Experimental results of horizontally specialised business model (Model 2)

\begin{tabular}{|l|l|l|l|l|}
\hline \multirow{2}{*}{} & \multicolumn{2}{|l|}{$(1.0,1.0)$} & \multicolumn{1}{l|}{$(0.5,1.0)$} \\
\cline { 2 - 5 } & Ave. & Std. Dev. & Ave. & Std. Dev. \\
\hline Profit & 6179.73 & 730.06 & 5919.06 & 708.20 \\
\hline Stock(WIP) & 21.28 & 2.57 & 21.82 & 2.68 \\
\hline Lead time & 16.44 & 2.14 & 13.47 & 1.81 \\
\hline & $(1.0,1.5)$ & \multicolumn{3}{|}{} \\
\cline { 2 - 5 } & Ave. & Std. Dev. & Ave. & Std. Dev. \\
\hline Profit & 9263.05 & 1084.19 & 8889.12 & 1047.26 \\
\hline Stock(WIP) & 32.12 & 3.82 & 33.04 & 3.81 \\
\hline Lead time & 25.49 & 2.99 & 20.67 & 2.59 \\
\hline
\end{tabular}

Table 4 Experimental results of hybrid model (Model 3)

\begin{tabular}{|l|l|l|l|l|}
\hline \multirow{2}{*}{} & $(1.0,1.0)$ & \multicolumn{3}{|c|}{$(0.5,1.0)$} \\
\cline { 2 - 5 } & Ave. & Std. Dev. & Ave. & Std. Dev. \\
\hline Profit & 7928.55 & 930.86 & 7628.55 & 930.86 \\
\hline Stock(WIP) & 22.32 & 2.70 & 22.32 & 2.70 \\
\hline Lead time & 16.69 & 2.07 & 12.98 & 1.62 \\
\hline & $(1.0,1.5)$ & \multicolumn{3}{|l}{} \\
\cline { 2 - 5 } & Ave. & Std. Dev. & Ave. & Std. Dev. \\
\hline Profit & 11877.20 & 1390.56 & 11877.20 & 1390.56 \\
\hline Stock(WIP) & 33.49 & 4.02 & 33.49 & 4.02 \\
\hline Lead time & 24.95 & 2.99 & 19.41 & 2.31 \\
\hline
\end{tabular}




\section{CONCLUSIONS}

In this paper, we focused on negotiation process in VE formation to clarify its effective management. We firstly classified business model into three types, such as vertically integrated business model, horizontally specialised business model and hybrid business model. Then we proposed CNP based negotiation protocol amongst enterprises with marketing science models, such as lexicographic model and MLH model, and N-person game theoretic approach. Marketing science based negotiation was applied into the vertically integrated business model considering a realistic enterprise management strategy. The horizontally specialised business model included $\mathrm{N}$-person game theoretic negotiations to realise the coordination amongst enterprises in the same business segment. We developed a computer simulation model to form VE through multiple negotiations amongst several potential members in the negotiation domain, and finally clarified the formation dynamism with the negotiation process. It has been confirmed that the vertically integrated business model is profit-oriented and it is the best in relatively stable business situations. On the contrary, horizontally specialised business model is robust against the order change, and it suits agile manufacturing situations. Hybrid business model is moderate characteristic between them, and it seems useful practically as often shown in real situations.

\section{REFERENCES}

1. Camarinha-Matos, L. M. et al, The virtual enterprise concept, Infrastructures for virtual enterprises, Kluwer academic publishers, Boston, pp.3-14, 1999.

2. Katahira H., Marketing science (in Japanese), Tokyo university press, Tokyo, 1987.

3. Von Neumann, J. et al., Theory of Games and Economic Behavior, Princeton University Press, 1947.

4. Smith, R., The contract net protocol, IEEE Transaction on Computers, C-29, pp.1104-1113, 1980.

5. Kaihara, T. and S. Fujii, A study on virtual enterprise coalition with multi-agent technology in agile manufacturing environment, International Journal of Advanced Manufacturing Systems, Vol.1, No.2, pp.125-139, 2002.

6. Kaihara, T. and S. Fujii, IT based Virtual Enterprise Coalition Strategy for Agile Manufacturing Environment, Proc. of the 35th CIRP Int. Seminar on Manufacturing Systems, pp 32-37, 2002.

7. Durfee, E. et al., Coherent cooperation among communication problem solvers, IEEE Transaction on Computers, N 36, pp.1275-1291, 1987.

8. Kansai Bureau of Economy, Trade and Industry, URL : http:/www.kansai.meti.go.jp/3-2sanki/clusterbeam/english.html. 